J. Phys. IV France 138 (2006) 13-19

(C) EDP Sciences, Les Ulis

DOI: 10.1051/jp4:2006138003

\title{
Laser XUV haute cadence pompé par laser Titane : Saphir, vers la station LASERIX
}

\author{
S. Kazamias ${ }^{1}$, K. Cassou ${ }^{1}$, D. Ros ${ }^{1}$, F. Plé ${ }^{1}$, G. Jamelot ${ }^{1}$, A. Klisnick ${ }^{1}$, \\ O. Lundh², F. Lindau², A. Persson ${ }^{2}$, C.-G. Wahlström² ${ }^{2}$ S. de Rossi ${ }^{3}$, \\ D. Joyeux ${ }^{3}$, B. Zielbauer ${ }^{4,5}$, D. Ursescu ${ }^{5}$ et T. Kühl ${ }^{5,6}$ \\ ${ }^{1}$ LIXAM, Laboratoire d'Interaction du Rayonnement $X$ avec la Matière, UMR 8624, \\ Bât. 350, Campus de l'Université Paris XI, 91405 Orsay Cedex, France \\ 2 Department of Physics, Lund Institute of Technology, PO Box 118, 22100 Lund, Sweden \\ ${ }^{3}$ LCFIO, Laboratoire Charles Fabry de I'Institut d'Optique, UMR 8501, Bât. 503, Campus de \\ I'Université Paris XI, 91405 Orsay Cedex, France \\ ${ }^{4}$ Max-Born Institut, Max-Born Strasse 2A, 12489 Berlin, Germany \\ ${ }^{5}$ Gesellschaft für Schwerionenforschung mbh, Planckstrasse 1, 64291 Darmstadt, Germany \\ ${ }^{6}$ Johannes-Gutenberg Universitat Mainz, Staudingerweg 7, 55128 Mainz, Germany
}

\begin{abstract}
Résumé. Nous présentons des résultats récents de laser $\mathrm{X}$ collisionnel transitoire pompé en incidence rasante à haut taux de répétition. Ils ont été obtenus à partir du laser de pompe Titane :Saphir $30 \mathrm{TW}$ disponible sur l'installation européenne du LLC à Lund (Suède). Nous avons démontré lors de cette expérience qu'il était possible d'obtenir en routine près de 3 microjoule par impulsion à $18,9 \mathrm{~nm}$ avec seulement $1 \mathrm{~J}$ d'énergie de pompe infrarouge. Nous avons plus particulièrement étudié l'influence de l'angle de rasance sur cible de l'impulsion laser qui vient pomper le plasma, quelques centaines de picosecondes après la première impulsion responsable de sa création. Un système d'imagerie XUV à haute résolution nous a en effet permis d'obtenir des informations précieuses sur la pupille de sortie du laser X, comme l'énergie totale dans la tache mais aussi la distance d'émission par rapport à la cible, et encore les dimensions horizontale et verticale de la source.
\end{abstract}

\section{INTRODUCTION}

Le présent travail s'inscrit dans une période charnière pour la recherche expérimentale et théorique dans le domaine des lasers X collisionnels générés par laser à partir de cibles solides. D'une part, cette thématique connaît depuis deux ans au niveau mondial de nouveaux développements depuis qu'une équipe américaine a démontré qu'il était possible de générer un rayonnement de type laser X à partir de seulement 1 Joule d'énergie de pompe, contre au minimum une dizaine de Joules jusque là [1]. Plus récemment, le fonctionnement de ce type de laser $\mathrm{X}$ en régime saturé a été prouvé expérimentalement par une autre équipe avec sensiblement le même niveau d'énergie de pompe [2]. Ce progrès a été permis grâce à une nouvelle technique de pompage de la cible en incidence rasante, appelée GRIP (Grazing Incidence Pumping en anglais) que nous détaillerons dans cet article. Compte tenu de la technologie sans cesse croissante des lasers de puissance à courte durée d'impulsion, et en particulier des chaînes d'amplification Titane :Saphir, ce résultat a permis de réaliser des sources laser X à des cadences de $10 \mathrm{~Hz}$, contre quelques tirs par heure pour les lasers $\mathrm{X}$ de la génération précédente [3]. Cette augmentation considérable de la cadence et également du nombre d'installations disponibles pour ce type d'expériences permet d'envisager des études expérimentales plus approfondies grâce à une analyse 
statistique. Les campagnes expérimentales sont également raccourcies et peuvent être plus nombreuses, ce qui contribue à stimuler la recherche internationale sur le sujet, comme le prouve le très grand nombre de publications parues cette année sur ce thème.

D'autre part, notre équipe s'est vue confier la responsabilité de la construction de la station LASERIX de l'Université Paris XI. Cette installation laser de puissance basée sur une chaîne d'amplification Titane : Saphir est entièrement destinée à la recherche sur les lasers X et leurs applications, ce qui constitue une première mondiale et une chance unique pour la recherche en France. Le travail présenté ici constitue une étape importante de validation des choix technologiques réalisés par l'équipe pour la conception de la source laser $\mathrm{X}$ de cette nouvelle installation qui sera opérationnelle courant 2007.

\section{LA PHYSIQUE DES LASERS X EN POMPAGE COLLISIONNEL TRANSITOIRE}

\subsection{Principe du laser XUV collisionnel transitoire}

Les lasers $\mathrm{X}$ sont des sources de lumière brillante dans la gamme de rayonnement de l'extrême UV : leurs longueurs d'onde sont comprises typiquement entre 5 et 50 nanomètres. Ces sources se caractérisent par une forte brillance spectrale pic due à leur grande monochromaticité (grande cohérence longitudinale), leur durée d'impulsion dans la gamme picoseconde et leur niveau d'énergie allant du microjoule au millijoule [4]. Ces sources présentent cependant jusqu'à présent une cohérence spatiale seulement partielle [5]. Les lasers $X$ sont issus de la création d'une inversion de population dans un plasma d'ions multichargés, de type nickelloïde dans notre cas. En régime collisionnel transitoire, ils sont obtenus à partir de l'irradiation laser d'une cible solide. Le pompage de la transition est réalisé par une deuxième impulsion très intense qui chauffe les électrons du plasma et provoque des collisions avec les ions, les faisant ainsi passer dans un niveau excité. Un laser X se comporte alors comme un système à trois niveaux dont le niveau intermédiaire se dépeuple rapidement par voie radiative vers le fondamental. On obtient ainsi l'inversion de population nécessaire à une émission laser. Dans notre expérience, nous avons étudié la raie à 18,9 $\mathrm{nm}$ dans le molybdène nickelloïde qui correspond à la transition $4 \mathrm{~d}->4 \mathrm{p}$. En fonctionnement ASE (amplified spontaneous emission en anglais), les premiers photons sont issus de l'émission spontanée en début de la colonne de plasma, puis le gain dans le milieu permet d'atteindre rapidement un régime d'émission stimulée.

\subsection{Particularité du pompage en incidence rasante}

En pratique, un laser $\mathrm{X}$ est réalisé par la superposition sur une cible solide de plusieurs mm de long de deux lignes focales laser de longueur supérieure à la cible et de largeur quelques dizaines de microns. La première impulsion, de durée supérieure à plusieurs centaines de picosecondes, est responsable de la création du plasma dans l'état d'ionisation requis. Elle attaque la cible en incidence normale. La seconde impulsion, de durée picoseconde, est absorbée quelques centaines de picosecondes plus tard dans le plasma par Bremsstrahlung inverse pour chauffer les électrons et créer ainsi une zone de gain. Dans la configuration GRIP, l'impulsion laser est incidente sur cible avec un angle de rasance compris typiquement entre 10 et $20^{\circ}$. Dans les expériences précédentes, elle arrivait sur la cible en incidence quasi normale. La différence fondamentale entre ces deux régimes est la densité à laquelle l'énergie est déposée dans le plasma : dans le cas de l'incidence normale, l'énergie est déposée au voisinage proche de la densité critique (notée $\mathrm{n}_{\mathrm{c}}$ ) alors qu'en configuration rasante, les rayons lumineux sont réfractés lors de leur traversée de milieux à gradients d'indice, cet effet est similaire à celui qui produit les mirages optiques. La densité maximale pouvant alors être atteinte par les rayons est donnée par la formule (1): $n=n_{c} \sin ^{2} \theta$, où $\theta$ est l'angle de rasance sur cible. On comprend alors que pour de faibles angles de rasance, la densité de la zone de gain sera plus faible que pour des angles plus élevés. Pour des angles importants, la densité du plasma sera forte mais présentera de forts gradients de densités électroniques 
qui pourront perturber la propagation du faisceau amplifié dans la zone de gain. L'angle optimal est donc un compromis entre ces deux phénomènes.

\section{DISPOSITIF EXPÉRIMENTAL}

Notre expérience laser X de pompage en incidence rasante s'est déroulée sur l'installation européenne du LLC (Lund Laser Centre) de l'Université de Lund en Suède. Nous avons utilisé la chaîne d'amplification Titane : saphir délivrant habituellement 30 TW à une cadence de $10 \mathrm{~Hz}$ par impulsions de 1 Joule en 30 femtosecondes. Pour les besoins de l'expérience, nous avons divisé en deux faisceaux l'énergie totale de 1,2 Joule à la sortie du dernier étage d'amplification, juste avant l'entrée dans le compresseur. Le premier faisceau, non comprimé, de durée 300 picosecondes et contenant environ $400 \mathrm{~mJ}$, a simplement été focalisé en ligne de dimension typique 70 microns par $6 \mathrm{~mm}$. Une ligne à retard et un générateur de pré-impulsion ont également été implantés sur cette ligne pour ajuster notamment le délai entre les deux impulsions. La deuxième impulsion est envoyée dans le compresseur pour réduire sa durée à 5 picosecondes. La ligne focale est ici réalisée par l'utilisation d'un simple miroir sphérique hors-axe (angle d'incidence compris entre 5 et $10^{\circ}$ ). Nous utilisons l'astigmatisme ainsi produit pour obtenir une ligne de dimension 40 microns par 4,2 mm. Les propriétés géométriques de cette ligne focale sont particulièrement intéressantes pour notre expérience car il se forme de façon naturelle une onde progressive intrinsèque se propageant à une vitesse proche de la vitesse de la lumière le long de la ligne. Ceci signifie que le front d'énergie déposée le long de la ligne focale de plasma se déplace à la vitesse de la lumière donc des photons XUV à amplifier. Nous ne sommes donc pas limités par la courte durée du gain laser X. Notre dispositif expérimental, représenté figure 1, nous permettait de changer sous vide l'angle de rasance sur cible tout en maintenant fixées la position et la direction des lignes focales. La superposition fine de ces dernières était vérifiée régulièrement grâce à un système d'imagerie à fort grandissement et de résolution micronique, amovible sous vide (RILF = reprise image de lignes focales).

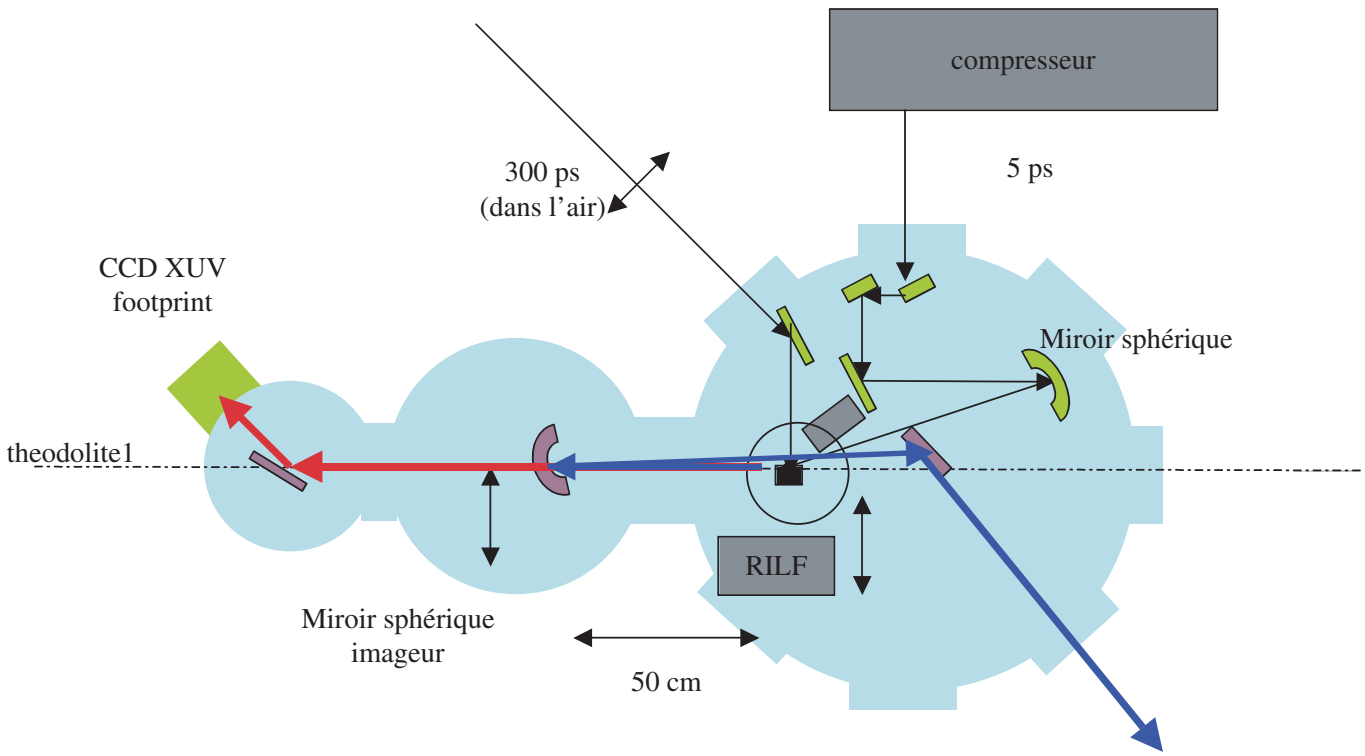

Vers CCD

Figure 1. Dispositif expérimental de l'expérience de pompage en incidence rasante. 


\section{DIAGNOSTIC DE LA SOURCE}

\subsection{Spectromètre champ plan}

Nous avons d'abord vérifié que la source laser $\mathrm{X}$ fonctionnait de façon fiable en observant l'émission XUV sur l'axe par un spectromètre à champ plan. Dans ce cas, la raie à $18,9 \mathrm{~nm}$ domine très nettement toutes les autres raies du plasma comme le montre la figure 2 .

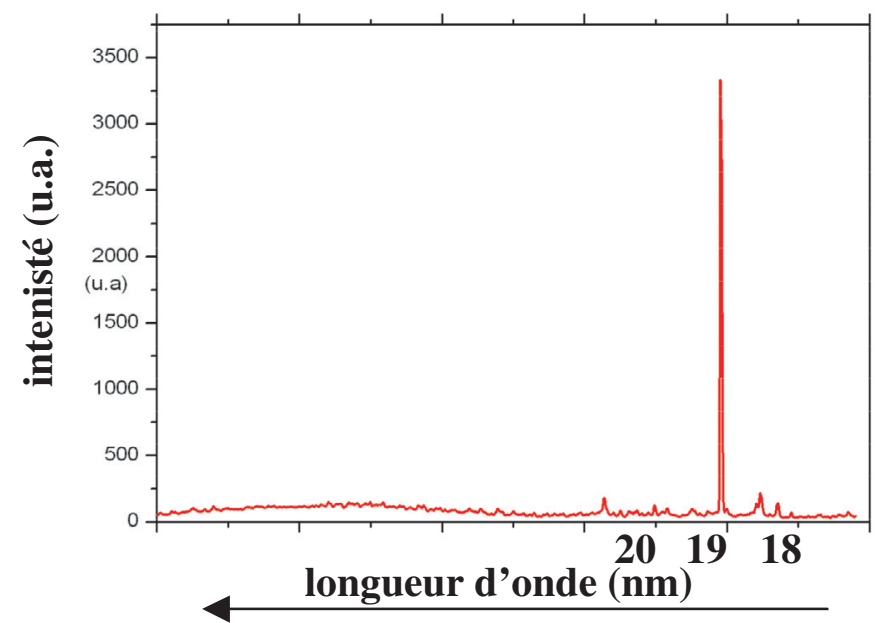

Figure 2. Spectre d'émission du plasma dans la gamme 10-20 nm en fonctionnement laser X.

\subsection{Imageur « champ proche»}

Les caractéristiques de la source laser $\mathrm{X}$ en fonction de différents paramètres, tels que le délai entre les deux impulsions et l'angle de rasance sur cible, ont été étudiées de façon approfondie grâce à un imageur haute résolution à la longueur d'onde du laser $\mathrm{X}$ de la pupille émettrice sur la face de sortie du plasma, appelé également « champ proche ». Il a été conçu et réalisé dans les ateliers du LCFIO de l'Institut d'Optique. L'optique utilisée est un miroir sphérique de focale $50 \mathrm{~cm}$ ayant reçu un traitement multicouche Mo : Si pour réaliser la sélection spectrale de la longueur d'onde du laser X et optimiser sa réflectivité. Les caractéristiques du dispositif sont un grandissement de 7,6 pour une CCD XUV ANDOR placée après environ 4 mètres du miroir. La résolution ainsi obtenue est de 1,7 micron dans le plan objet, ce qui correspond à 1 pixel de la caméra. Cette dernière était protégée de tout rayonnement parasite issu du plasma ou de réflexions du laser infrarouge par un filtre d'aluminium dont l'épaisseur était comprise entre 2 et 6 microns, suivant l'énergie XUV dans la source. Une simple image de champ proche nous permet de déterminer non seulement l'énergie intégrée de la source mais aussi ses dimensions horizontale et verticale, de même que sa position par rapport à la surface de la cible.

\section{RÉSULTATS EXPÉRIMENTAUX}

\subsection{Caractéristiques de la source}

La figure 3 présente une des images les plus intenses obtenues lors de notre expérience dans le cas d'un angle de rasance sur cible de $19^{\circ}$ et pour un délai entre les deux impulsions laser de $400 \mathrm{ps}$. L'énergie intégrée dans la tache atteint 3 microjoules et cette efficacité importante a été obtenue en routine avec une bonne reproductibilité statistique, de l'ordre de $10 \%$. Les dimensions caractéristiques 


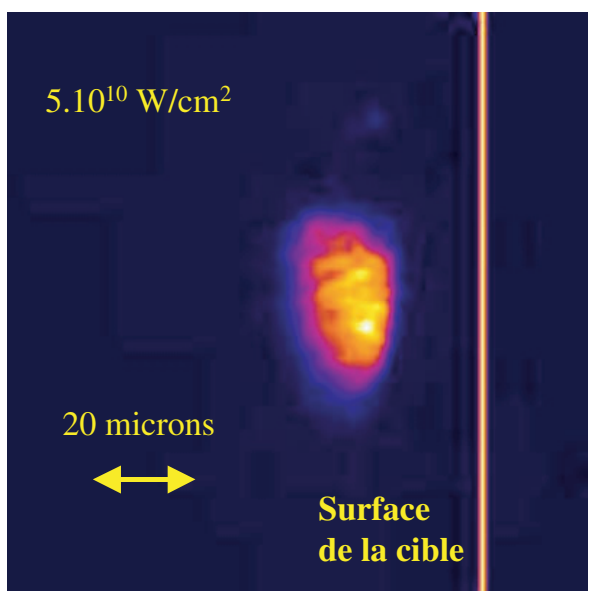

Figure 3. Allure caractéristique de la pupille de sortie du plasma en fonctionnement laser X.

de la source sont 20 microns dans la direction horizontale et 40 microns pour la direction verticale, tandis que la position d'émission est à 35 microns de la surface de la cible. En supposant une durée d'émission de 5 ps [3], l'éclairement intégré à la source atteindrait ainsi $5.10^{10} \mathrm{~W} / \mathrm{cm}^{2}$. Cette valeur élevée nous indique que le laser $\mathrm{X}$ ci-dessus fonctionne en régime saturé. Nous avons par ailleurs trouvé, en modifiant notamment les caractéristiques de la pré-impulsion et de la balance d'énergie entre les deux impulsions, un autre régime de fonctionnement pour lequel la tache contenait autant d'énergie mais avait une forme plus symétrique et une taille caractéristique de 10 microns. Dans ce cas, des éclairements proches de $10^{11} \mathrm{~W} / \mathrm{cm}^{2}$ ont été atteints, ce qui en fait une source très brillante, particulièrement adaptée aux applications interférométriques par exemple [6].

\subsection{Influence de l'angle de rasance}

Un des objectifs principaux de notre campagne expérimentale était de comprendre le rôle de l'angle de rasance sur cible. Les résultats sont représentés sur la figure 4 pour des angles variant de 15 à $21^{\circ}$.

Nous observons un maximum d'émission très net pour des angles compris entre 17 et $19^{\circ}$. L'énergie intégrée dans la source chute d'environ un ordre de grandeur pour les autres valeurs d'angle, de part et

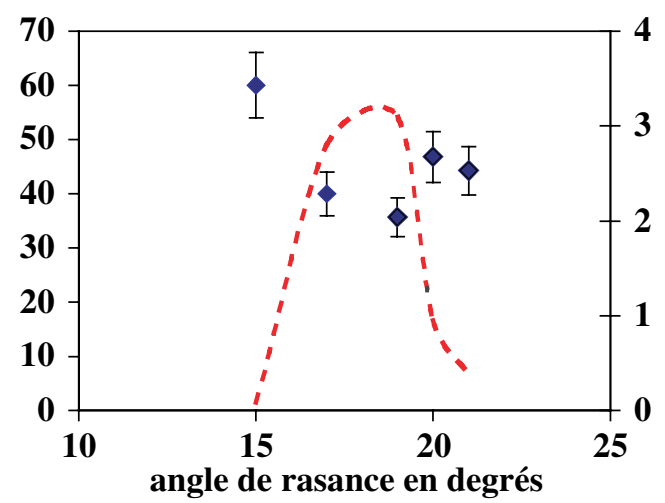

Figure 4. Variation de l'énergie émise par la source en microjoule sur l'axe de droite (pointillés) et de la distance d'émission à la cible (losanges) en fonction de l'angle de rasance sur cible. 
d'autre du maximum. La position de la pupille de sortie du laser X est très instructive sur ce qui se passe lors de l'amplification dans la colonne du plasma : nous voyons que le maximum d'énergie produite correspond à la tache la plus près possible de la cible. Pour des angles de rasance inférieurs à $19^{\circ}$, cette distance décroît avec l'angle comme le prédit logiquement la formule (1) car les zones de densité les plus fortes sont plus près de la surface initiale de la cible. Pour des angles croissants, en l'absence de problème de propagation pour le faisceau amplifié, la zone d'émission se rapproche nettement de la cible car l'énergie de pompe est déposée dans des zones de plus en plus denses du plasma, ce qui favorise le gain laser X donc augmente l'énergie produite. Nous attribuons le comportement ultérieur de la courbe à des problèmes de réfraction du faisceau laser $\mathrm{X}$ en raison de forts gradients de densité électronique qui ont pour effet de modifier la trajectoire des photons amplifiés dans le plasma, et de les faire progressivement sortir des zones à fort gain proches de la cible.

\subsection{Fonctionnement à $10 \mathrm{~Hz}$}

Nous avons réalisé des films montrant le fonctionnement à $10 \mathrm{~Hz}$ de la source laser $\mathrm{X}$ en régime saturé. Ils permettent de démontrer une très bonne stabilité de pointé due essentiellement à la bonne stabilité initiale du laser infrarouge de pompe : la source laser X ne fluctue pas plus que sa propre taille. Elle est naturellement plus sensible aux fluctuations du laser dans la direction verticale qu'horizontale. Nous remarquons cependant une dégradation du faisceau et un creusement progressif de la cible solide lorsqu' on l'irradie toujours à la même position, pendant plus de 50 à 100 tirs. Cela signifie qu'à $10 \mathrm{~Hz}$ il conviendrait de tirer sur une cible déroulante présentant une surface vierge environ toutes les 3 à 5 secondes. La mise en place d'un tel dispositif est prévue sur la station LASERIX.

\subsection{Allure des champs lointains}

Un champ lointain, appelé également footprint, est une empreinte de faisceau obtenue sur une caméra CCD XUV après une certaine distance de propagation libre depuis la source, constituée ici par la face de sortie du plasma appelée également champ proche. Les images obtenues sont très instructives car elles permettent de mesurer l'angle d'émission du faisceau laser $\mathrm{X}$ et de mesurer sa divergence. Nous avons évalué cette divergence à environ $5 \mathrm{mrad}$ et estimons que pour l'angle de rasance $19^{\circ}$, le faisceau était émis pratiquement sur l'axe des lignes focales, c'est-à-dire sans réfraction à l'intérieur du plasma.

Ces images apportent également des informations précieuses sur les caractéristiques de cohérence initiale de la source, comme cela est démontré dans la thèse d' Olivier Guilbaud et dans la référence [5]. Les images présentées figure 5 correspondent à une distance de propagation de 1,5 mètre.

Dans le cas où la source initiale est composée d'une seule tache, l'allure du champ lointain montre un faisceau avec une allure de speckle. Ceci est dû à la grande monochromaticité de la source, alliée à une courte durée d'impulsion et une faible cohérence spatiale. Dans le cas où l'on est capable de faire apparaître deux sources distinctes dans la direction verticale en jouant sur le délai entre les deux impulsions et la taille des lignes focales, on voit apparaître dans le champ lointain des franges horizontales qui résultent de l'interférence entre ces deux sources. L'étude précise de ces franges permet de remonter aux caractéristiques du champ proche et notamment à ses dimensions [5].

\section{CONCLUSION}

Lors de notre dernière campagne expérimentale au LLC (Lund Laser Centre), nous avons démontré la possibilité de générer, à 18,9 nm de longueur d'onde, une source laser XUV collisionnelle transitoire pompée en incidence rasante, avec seulement 1 Joule d'énergie de pompe et une cadence de $10 \mathrm{~Hz}$. L'efficacité de conversion obtenue atteint $3 \cdot 10^{-6}$ pour un angle de rasance sur cible de $19^{\circ}$, ce qui en fait à notre connaissance une des sources laser $\mathrm{X}$ les plus efficaces démontrées à ce jour. De plus, la stabilité 

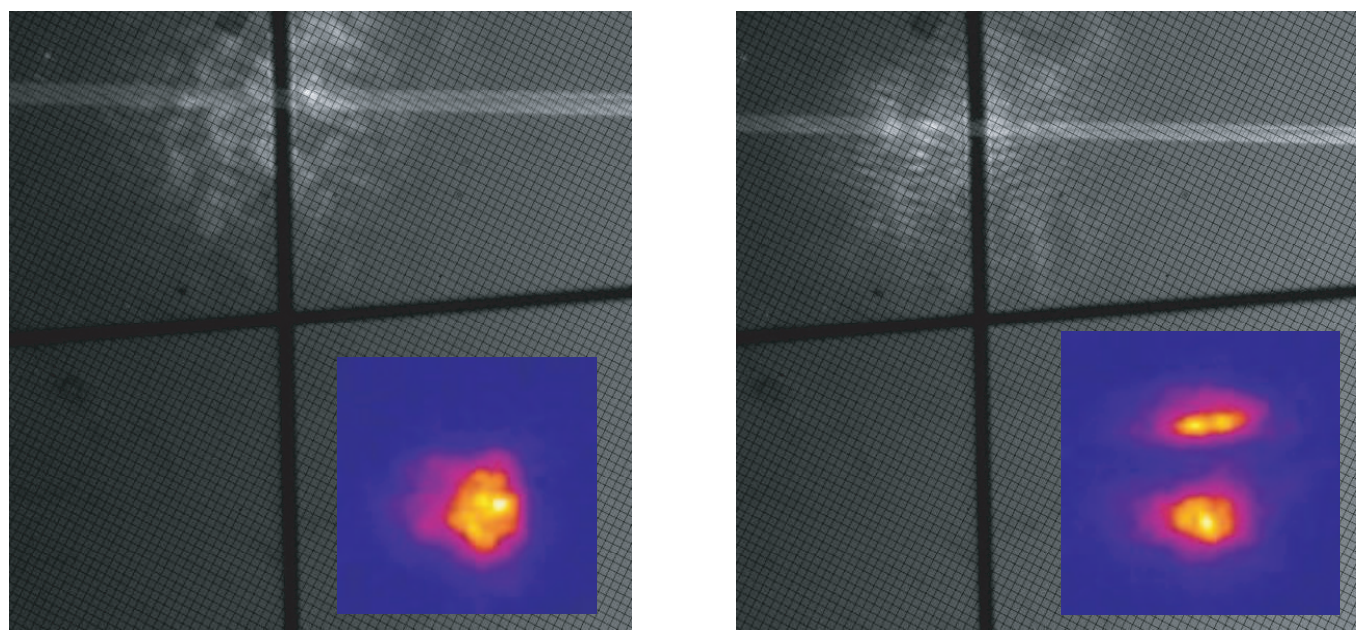

Figure 5. Allure du champ lointain du faisceau laser $X$ pour deux allures typiques du champ proche.

de la source, tant en énergie qu'en pointé en fait une source déjà intéressante pour des applications ultérieures.

Compte tenu de l'énergie de pompe qui sera dès le début 2007 disponible sur la station laser X, nous prévoyons avec le même schéma de pompage, de pouvoir d'une part atteindre des longueurs d'onde autour de $10 \mathrm{~nm}$ et d'autre part de fournir aux utilisateurs une source de plusieurs dizaines de microjoule par impulsion à $0,1 \mathrm{~Hz}$ ou de quelques microjoule à $10 \mathrm{~Hz}$.

\section{Remerciements}

Cette expérience a été soutenue par le programme européen LASERLAB d'accès aux installations laser (contrat RII3-CT-2003-506350, Laserlab Europe).

\section{Références}

[1] R. Keenan, J. Dunn, P.K. Patel, D.F. Price, R.F. Smith, V.N. Shlyapsev, Phys. Rev. Lett. 94, 103901 (2005)

[2] B.M. Luther, Y. Wang, M.A. Larotonda, D. Alessi, M. Berrill, M.C. Marconi, J.J. Rocca, Opt. Lett. 30, 165 (2005)

[3] A. Klisnick, O. Guilbaud, D. Ros, K. Cassou, S. Kazamias, G. Jamelot, J.C. Lagron, D. Joyeux, D. Phalippou, Y. Lechantre, M. Edwards, P. Mistry, G.J. Tallents, JQSRT 99, 370 (2006)

[4] B. Rus, T. Mocek, A.R. Prag, M. Koslova, G. Jamelot, A. Carillon, D. Ros, D. Joyeux, D. Phalippou, Phys. Rev. A 66, 063805 (2002)

[5] O. Guilbaud, A. Klisnick, K. Cassou, S. Kazamias, D. Ros, G. Jamelot, D. Joyeux and D. Phalippou, Europhys. Lett., 74 (5), pp. 823-829 (2006)

[6] K. Cassou et al., S. de Rossi et al., dans le présent ouvrage 